\title{
Nanoscale Conducting Oxide PlasMOStor
}

\author{
Ho W. Lee, ${ }^{*}, \dagger, \ddagger$ Georgia Papadakis, ${ }^{\dagger}$ Stanley P. Burgos, ${ }^{\dagger, \dagger}$ Krishnan Chander, $^{\dagger}$ Arian Kriesch, ${ }^{\dagger, \S}$ \\ Ragip Pala, ${ }^{\dagger, \ddagger}$ Ulf Peschel, ${ }^{\S}$ and Harry A. Atwater $*,+$,
}

${ }^{\dagger}$ Thomas J. Watson Laboratories of Applied Physics, California Institute of Technology, Pasadena, California 91125, United States

${ }^{\ddagger}$ Kavli Nanoscience Institute, California Institute of Technology, Pasadena, California 91125, United States

${ }^{\S}$ Institute of Optics, Information and Photonics and Graduate School in Advanced Optical Technologies, Friedrich-Alexander-University Erlangen-Nuremberg, 91054 Erlangen, Germany

\section{Supporting Information}

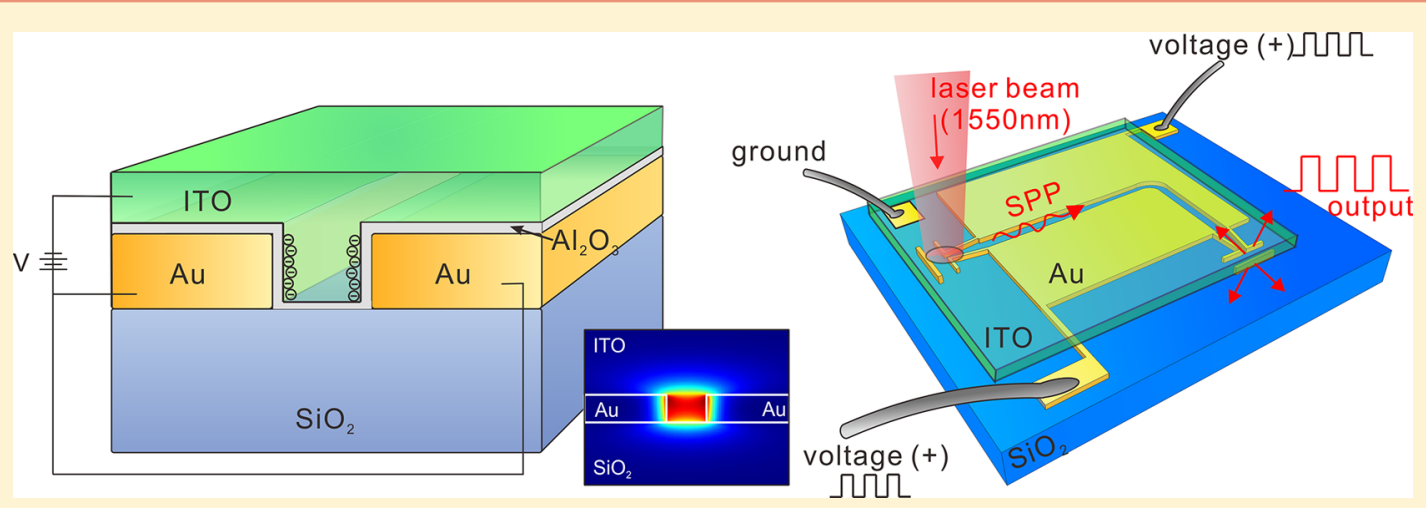

ABSTRACT: We experimentally demonstrate an ultracompact PlasMOStor, a plasmon slot waveguide field-effect modulator based on a transparent conducting oxide active region. By electrically modulating the conducting oxide material deposited into the gaps of highly confined plasmonic slot waveguides, we demonstrate field-effect dynamics giving rise to modulation with high dynamic range $(2.71 \mathrm{~dB} / \mu \mathrm{m})$ and low waveguide loss $(\sim 0.45 \mathrm{~dB} / \mu \mathrm{m})$. The large modulation strength is due to the large change in complex dielectric function when the signal wavelength approaches the surface plasmon resonance in the voltage-tuned conducting oxide accumulation layer. The results provide insight about the design of ultracompact, nanoscale modulators for future integrated nanophotonic circuits.

KEYWORDS: Plasmonics, transparent conducting oxide, modulator, field-effect modulation, epsilon near-zero material, nanocircuits, plasmonic slot waveguide, active plasmonics

$\mathrm{W}$ ith critical dimensions of transistors and chip-based photodetectors approaching a few nanometers, there remains a significant mismatch between the surface area, mode volume, and power density of integrated electronic devices and that of Si-based photonic components, which have characteristic dimensions in the hundreds of nanometer to micrometer range. Plasmonic active components can provide a capability for active modulation of lightwave signals in interconnects between future electronic and photonic networks co-located on a chip, since plasmonic structures can guide light in small modal volumes while maintaining a high optical bandwidth. ${ }^{1-7}$ Plasmonic structures also enable facile integration of electrical gates and contacts, allowing researchers to realize ultracompact active optical components such as optical transistors, photodetectors, and modulators as candidate components for nextgeneration integrated lightwave circuits.

The design of a plasmonic modulator for integrated photonic network applications has several important criteria: (1) a high on-off ratio ( $>3 \mathrm{~dB}),(2)$ low insertion loss $(<1 \mathrm{~dB}),(3)$ low energy consumption $(<10 \mathrm{fJ} / \mathrm{bit})$, (4) high operation speed
( $>$ few ten's of $\mathrm{GHz}$ ), and (5) small device dimensions $(<5$ $\mu \mathrm{m}){ }^{2,4,8}$ A variety of experimental reports have demonstrated plasmonic modulation based on different active tuning mechanisms. $^{9-18}$ However, in most cases the reported modulation concept either requires a large device areal footprint, a high bias voltage, or exhibits high photonic insertion losses. Several theoretical reports have also proposed efficient modulation mechanisms, but so far there has been no unequivocal demonstration of these concepts. ${ }^{19-26}$

The "PlasMOStor" ${ }^{10}$ represented the first electrically driven plasmonic modulator based on carrier-induced index changes in a metal-oxide-semiconductor (MOS) configuration, with the semiconductor driven into accumulation at low voltages and power densities $\left(\sim 10^{-7} \mathrm{~W} / \mathrm{cm}^{2}\right)$ owing to the presence of a thin insulating gate dielectric separating the active channel, which also forms a high confinement waveguide section. The

Received: August 5, 2014

Revised: October 3, 2014

Published: October 10, 2014 


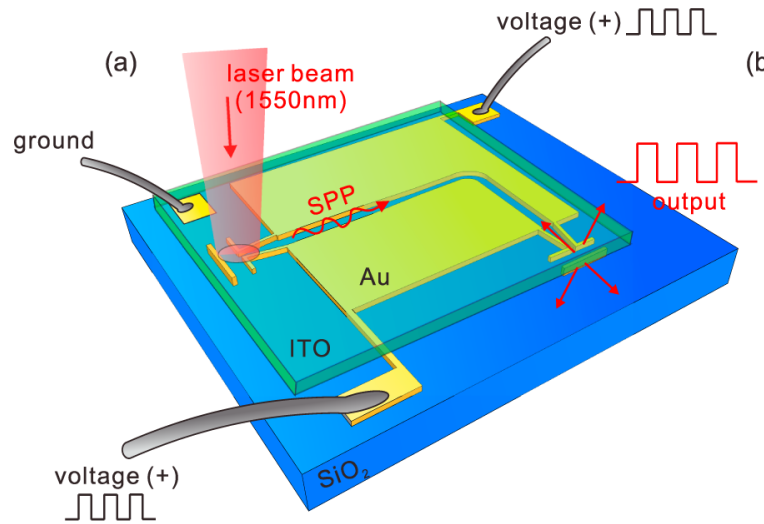

(b)

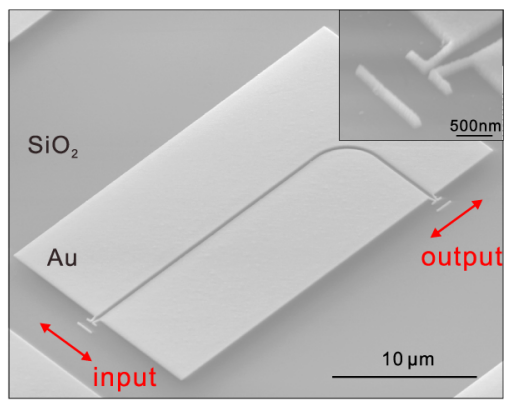

(c)

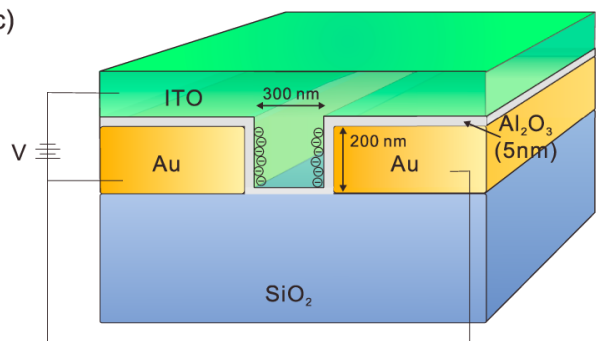

(e)

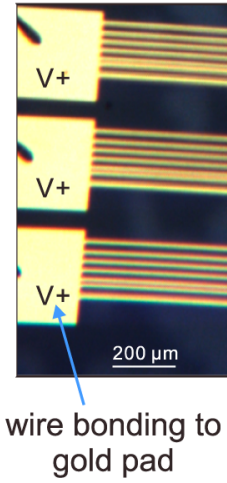

(d)

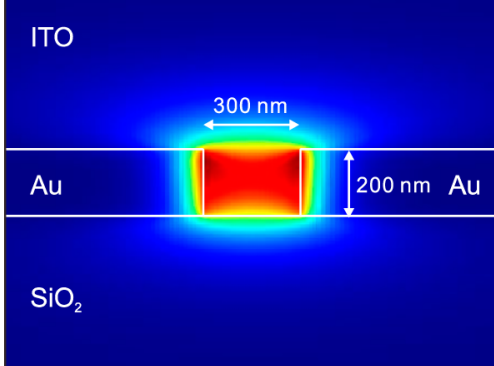

(f)

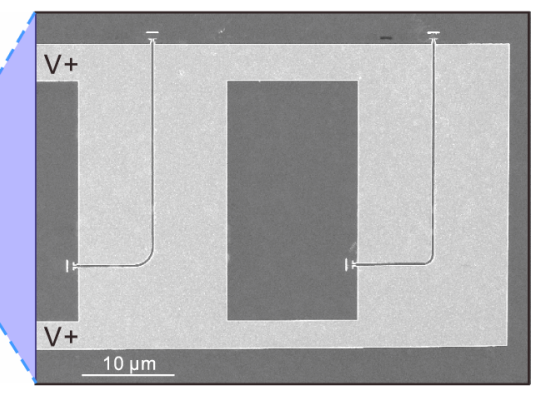

Figure 1. (a) Schematic of an antenna-coupled plasmonic slot waveguide modulator. (b) Scanning electron micrograph of plasmonic slot waveguide with nano-antenna. Inset: enlarged image of the nano-antenna. (c) Schematic of ITO-filled plasmonic slot waveguide showing a formation of accumulation layer in the interface of $\mathrm{Al}_{2} \mathrm{O}_{3} / \mathrm{ITO}$ under bias. (d) Finite element modeling of the magnetic field distributions for the corresponding gap-plasmon mode. (e) Microscope image of the modulator structure with external gold pad for wire connection. The waveguide array (right side) is filled with $5 \mathrm{~nm} \mathrm{Al}_{2} \mathrm{O}_{3}$ and $300 \mathrm{~nm}$ of ITO. (f) SEM image of the connected waveguides.

Si-based plasMOStor opened the opportunity for electrically driven on-chip devices. However, in that device, the observed refractive index changes under carrier accumulation were small due to the inherently low charge carrier concentrations $\left(10^{15}-\right.$ $10^{17} \mathrm{~cm}^{-3}$ ) in the quasi-neutral bulk portion of the silicon active region. ${ }^{10}$

By contrast, higher charge carrier concentrations can be achieved in transparent conducting oxide active regions since conducting oxides can be heavily doped with typical quasineutral region charge carrier concentrations between $10^{19}-10^{21}$ $\mathrm{cm}^{-3}$, allowing for plasma frequencies in the near-infrared and visible spectral range (see Supporting Information for details). ${ }^{27}$ These conducting oxide active regions have high transmittance at visible and near-infrared frequencies but can exhibit metallic characteristics (e.g., negative permittivity) in the near-infrared if sufficiently heavily doped. ${ }^{28,29}$ The conducting oxide carrier density at the gate dielectric/ conducting oxide interface can be modulated under an applied bias, similar to Si-based field-effect MOS devices where an accumulation layer is formed under an applied gate bias. In the case of conducting oxides, this accumulation layer induces a large change in the local complex index of refraction. A unityorder refractive index change $(\Delta n=1.39)$ was reported in a metal-oxide-conducting oxide heterostructure excited at a free space wavelength of $800 \mathrm{~nm} .{ }^{30}$ We note this refractive index change is at least 2 orders of magnitude higher than is seen for electro-optic modulation using the Kerr effect where the maximum refractive index change $\Delta n$ is on the order of $\sim 10^{-2}$. Because of the high modal confinement in plasmonic waveguides, integrating conducting oxides can lead to large changes in the plasmonic mode effective index via field-effect modulation, small active region areas, low energy consumption, and low insertion loss. ${ }^{30}$ To date, several attempts have been made to realize an efficient conducting oxide-based plasmonic modulator, but reported modulation strengths were relatively low, with an on-off ratio of $\sim 1 \mathrm{~dB} / \mu \mathrm{m}$. ${ }^{9,14}$ This relatively 

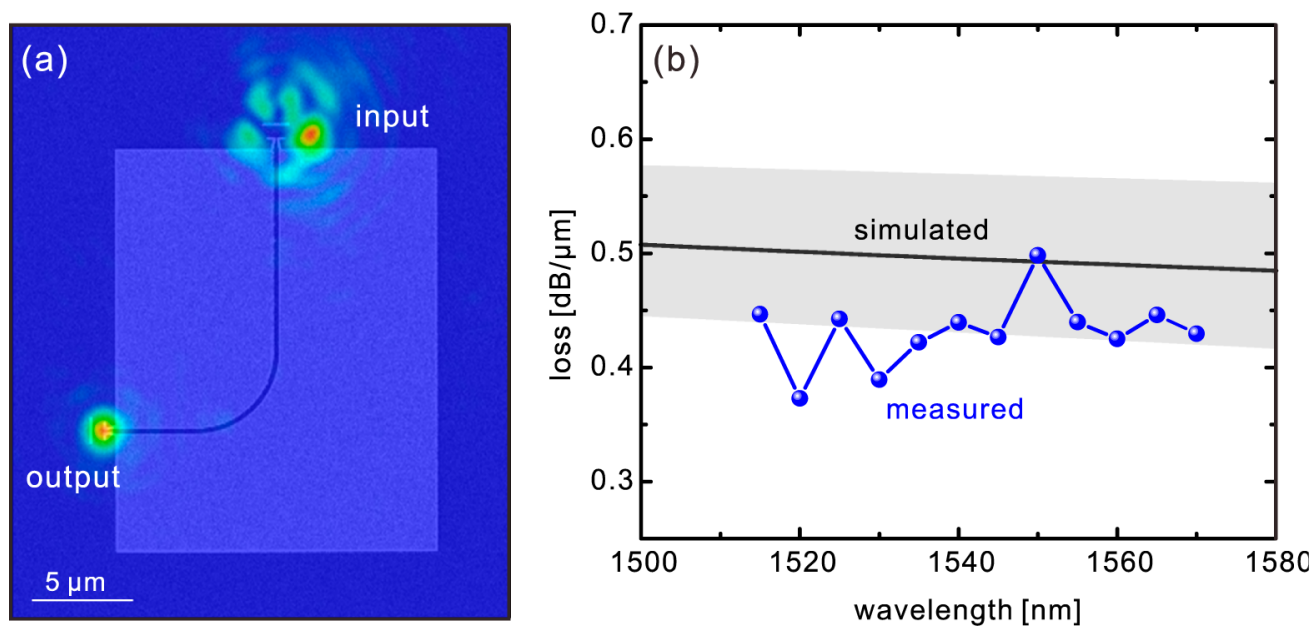

Figure 2. (a) Optical far-field image of an ITO-filled plasmonic slot waveguide at $\lambda_{0}=1550 \mathrm{~nm}$, superimposed onto a SEM of the structure. (b) Measured and simulated loss spectrum of the ITO-filled slot waveguide for a carrier concentration of ITO $n=1.6 \times 10^{19} \mathrm{~cm}^{-3}\left(\mathrm{shaded}_{\text {areas indicate }}\right.$ the results with $n \pm 1 \times 10^{19} \mathrm{~cm}^{-3}$ variation on the carrier concentration of the ITO).

modest modulation is mainly due to the weak overlap between the hybrid plasmonic modes used and the nanometer-size thin accumulation layer in the conducting oxide layer. Several theoretical reports have proposed mechanisms to enhance modulation strength by, e.g., introducing a nanotaper and an epsilon-near-zero material into the modulator design. ${ }^{31-34}$

The first element of our device design involves integration of conducting oxides into antenna-coupled plasmonic slot waveguides, ${ }^{7}$ as depicted in Figure 1. First we determined that the loss of indium tin oxide (ITO)-filled slot plasmonic waveguides was $\sim 0.45 \mathrm{~dB} / \mu \mathrm{m}$ at zero bias using far-field microscopy. We next measured a modulation of $2.71 \mathrm{~dB} / \mu \mathrm{m}$ by applying a $2.2 \mathrm{~V}$ bias in such an ITO-filled plasmonic waveguide, indicating that a micron-length device could achieve $3 \mathrm{~dB}$ modulation at $\lambda_{0}=$ $1550 \mathrm{~nm}$. We find that the largest modulation occurs when the ITO complex refractive index gives rise to a plasmon resonance near the signal wavelength.

Yagi-style optical antennas allow for efficient light coupling from free-space into the slot waveguides (15/45\% coupling efficiency from air/silica for silica cladded $\mathrm{Au}$ waveguides), which exhibit low propagation loss (propagation length of $\sim 22$ $\mu \mathrm{m}$ at $\left.\lambda_{0}=1550 \mathrm{~nm}\right) .^{7}$ Figure $1 \mathrm{~b}$ shows an example of the structure fabricated with electron-beam lithography patterning of a $200 \mathrm{~nm}$ Au layer on silica to form a waveguide slot width of $300 \mathrm{~nm}$ (see Methods below for fabrication details). To realize a field-effect modulator, a thin layer $(5 \mathrm{~nm})$ of aluminum oxide was deposited to form an insulating gate dielectric, deposited by atomic layer deposition which typically provides pinhole-free layers at thicknesses as small as $\sim 1 \mathrm{~nm}$, depending on the surface condition of the host material. ${ }^{35}$ By applying a voltage between the $\mathrm{Au}$ and the ITO, an accumulation layer formed at the $\mathrm{Al}_{2} \mathrm{O}_{3}-\mathrm{ITO}$ interface, modifying the complex refractive index of the propagating mode inside the gap since there exists a strong overlap between the field peak intensity of the plasmonic mode and the electron accumulation layer in the ITO (Figure 1c,d). Figure 1e shows an optical microscope image of the waveguide array structure with external gold pads for wire connections (left), and the array (right) is filled with $\mathrm{Al}_{2} \mathrm{O}_{3}$ and ITO. The SEM image of the connected waveguides is depicted in Figure 1f.

The optical characteristics of the propagating gap plasmon were measured with a cross-polarized far-field imaging configuration (see Methods below and Supporting Information for the detail of measurement). ${ }^{7,36}$ A typical measured image of an ITO-filled slot waveguide is displayed in Figure 2a, showing a clear output signal with good transmission. We directly measured the loss of the ITO-filled waveguide by measuring the transmitted spectra for different waveguide lengths of 35.28, $30.28,25.28,10.28 \mu \mathrm{m}$. Fitting the data at each wavelength to an exponentially decaying function resulted in the attenuation spectrum represented by dotted data in Figure $2 \mathrm{~b}$. As shown in the figure, the measured loss at zero bias of the conducting oxide-based plasmonic waveguide is $\sim 0.45 \mathrm{~dB} / \mu \mathrm{m}$ at telecommunication wavelengths $\left(\lambda_{0}=1520-1570 \mathrm{~nm}\right)$, providing sufficient transmission to realize nanocircuit devices. Compared with the loss of a $\mathrm{SiO}_{2}$-filled slot waveguide $(\sim 0.25 \mathrm{~dB} / \mu \mathrm{m})$, the ITO-filled waveguide exhibits several times higher propagation loss, due to the intrinsic material loss caused by the high ITO carrier concentration. However, it should be noted that with a potential device length less than a few micrometers, the propagation loss is not the foremost challenge for designing a very compact high-efficiency modulator as compared with achieving low insertion loss. Furthermore, the propagation loss could be further reduced by optimizing the plasmon slot structure, by coupling very short active region segments to dielectric waveguides (via a more complex fabrication process than is demonstrated here), or by using other lower loss conducting oxide materials. ${ }^{28}$ The modal structure and propagation were modeled using full-wave electromagnetic simulations (FDTD Solutions by Lumerical, Inc.), which included the complex frequency-dependent permittivity of gold $^{37}$ together with the standard Sellmeier model for silica. ${ }^{38}$ The complex permittivity of ITO was calculated using the standard free-electron Drude model (see Supporting Information for detail), ${ }^{39}$ with a charge carrier concentration of $n=1.6 \times 10^{19} \mathrm{~cm}^{-3}$, which was obtained from Hall effect measurements. ${ }^{40,41}$ The complex propagation constant and the loss of the gap-plasmonic mode of the ITOfilled slot waveguide were simulated. As seen in Figure $2 b$, the simulated waveguide loss of $\sim 0.45 \mathrm{~dB} / \mu \mathrm{m}$ agrees quite well with the measured value. The slight discrepancy may be attributed to the small geometry and dielectric constant difference between the simulated and experimental structures. 
To investigate the modulation properties of conducting oxide-filled plasmonic slot waveguides, structures were fabricated with different waveguide lengths and external gold pads for electrical contacts. Far-field images were taken while applying a gate bias between the ITO and gold electrodes (Figure 1e,f). As shown in Figure $3 a-c$, waveguide transmission
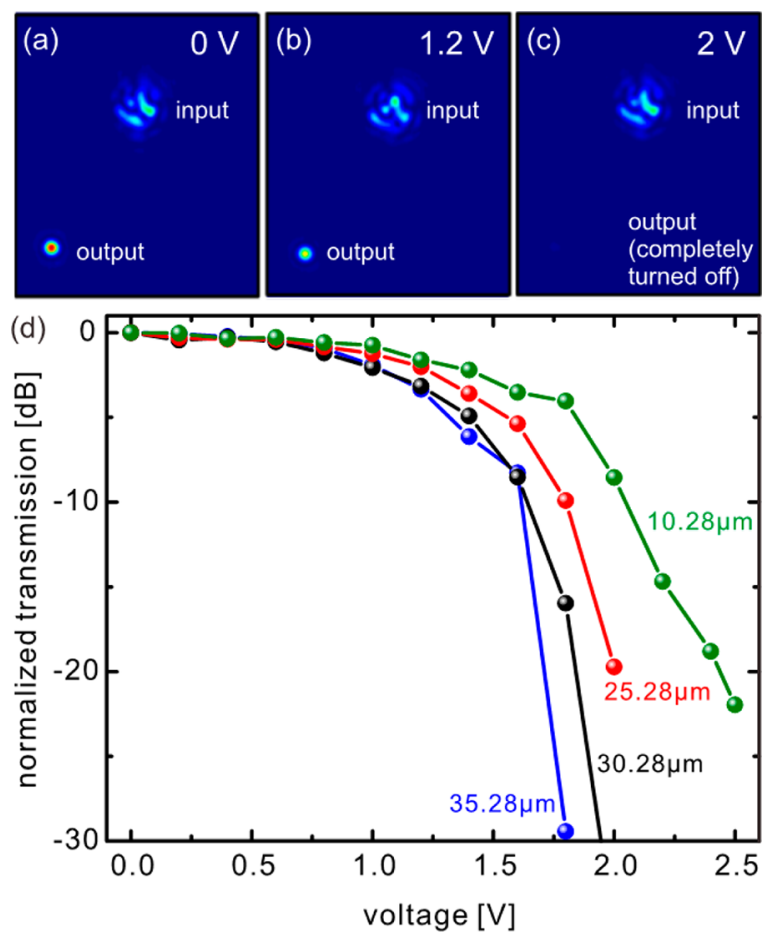

Figure 3. Measured optical far-field images of the ITO modulator with applied voltage of (a) 0 , (b) 1.2 , and (c) $2 \mathrm{~V}$ at $\lambda=1.55 \mu \mathrm{m}$. (d) The normalized transmission with the applied voltages for four different waveguides with different lengths.

is significantly reduced with an applied voltage of $\sim 1.2 \mathrm{~V}$ at a free space wavelength of $\lambda_{0}=1550 \mathrm{~nm}$. Transmission is almost completely turned off with an applied voltage of $2 \mathrm{~V}$. We measured the voltage-dependent transmission for four different waveguide lengths, and the results are shown in Figure $3 \mathrm{~d}$. Waveguide transmission was normalized to the transmission of an unbiased waveguide of the corresponding length for comparison. It is clear that the long waveguide $(35.28 \mu \mathrm{m})$ (e.g., blue curve in Figure 3d) required low voltage of $\sim 1.6 \mathrm{~V}$ to reduce the output intensity by $-20 \mathrm{~dB}$, while the short waveguide $(10.28 \mu \mathrm{m})$ (e.g., green curve) required around 2.4 $\mathrm{V}$ to drop the intensity level by $-20 \mathrm{~dB}$. We note that the $5 \mathrm{~nm}$ $\mathrm{Al}_{2} \mathrm{O}_{3}$ layer exhibits electrical breakdown at $\sim 2.3-2.5 \mathrm{~V}$, which is consistent with observations for $\mathrm{Al}_{2} \mathrm{O}_{3}$ layers grown by atomic layer deposition (see Supporting Information for detail). ${ }^{42}$ Higher breakdown voltages could potentially be achieved by replacing the $\mathrm{Al}_{2} \mathrm{O}_{3}$ dielectric layer with a material exhibiting higher DC permittivity, such as $\mathrm{HfO}_{2}\left(\varepsilon_{\mathrm{r}}=25\right){ }^{42}$ From the data in Figure 3, the change in modulation strength with applied voltage at $1550 \mathrm{~nm}$ wavelength was calculated (Figure $4 \mathrm{a}$, blue curve). A record high modulation strength of $\sim 2.71 \mathrm{~dB} / \mu \mathrm{m}$ is observed at a $2.2 \mathrm{~V}$, showing the potential of conducting oxide-based plasmonic modulators for next generation optical networks. Comparing with other reported plasmonic modulators, such as $\mathrm{VO}_{2}$-based plasmonic waveguide modulators $(2.3 \mathrm{~dB} / \mu \mathrm{m})^{13}$ and conducting oxide hybrid $\mathrm{Si}$ / plasmonic waveguide induced modulators $(1 \mathrm{~dB} / \mu \mathrm{m}),{ }^{14}$ our device shows the highest modulation strength, attributable to both the strong tunability of conducting oxides and the high field confinement of plasmonic slot waveguides. The experiment was repeated using thicker $\mathrm{Al}_{2} \mathrm{O}_{3}$ layers (thickness $=20$ $\mathrm{nm}$ ), but we did not observe any significant change in transmission until dielectric breakdown, further indicating the importance of using a thin high- $k$ insulator layer to maximize the conducting oxide accumulation-layer-induced modulation. It should be noted that the modulation mechanism in the current device is based on field-effect carrier-induced absorption and does not rely on the change of the real part of the mode index. However, one could also design a device exploiting voltage-induced phase change (real part of refractive index) to realize an efficient modulation scheme, such as using a stub or ring resonator, where the phase plays an important role in phase modulation dynamics.
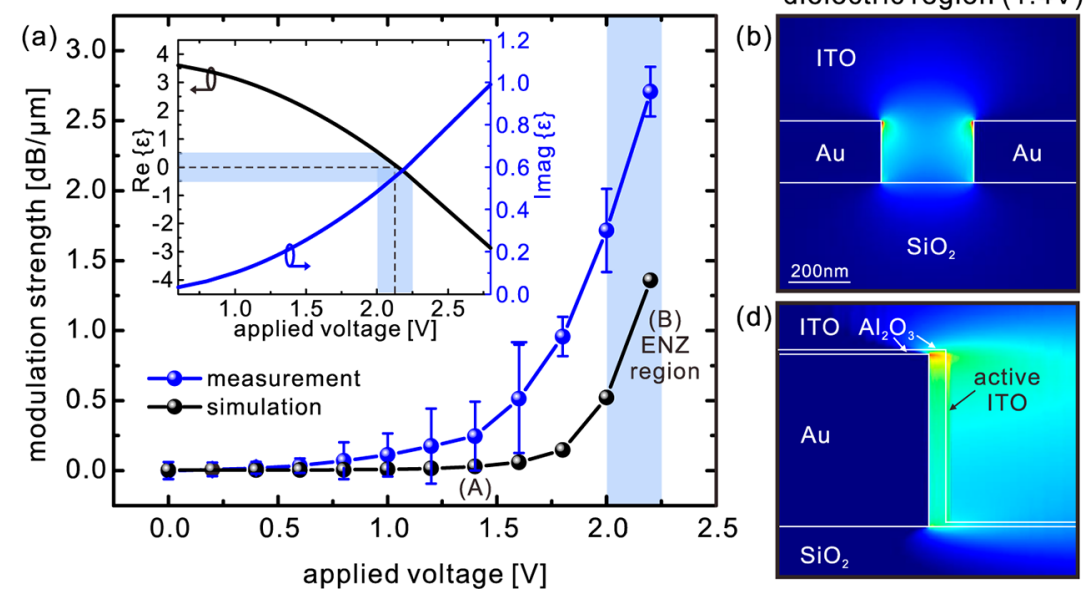

ENZ region $(2.2 \mathrm{~V})$

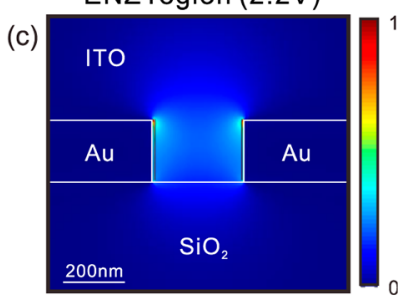

(e)

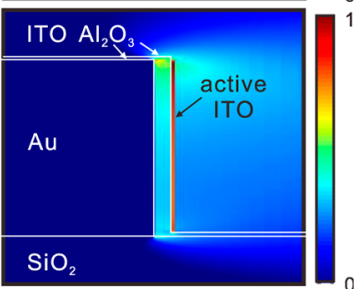

Figure 4. (a) The change of the measured (blue) and simulated (black) modulation strengths with the applied voltages. Inset: Corresponding complex permittivity of the ITO active layer at different applied voltages. Simulated mode field profile for (b) modulated layer with positive epsilon (A) (e.g., $\varepsilon_{\text {real }}=2.4,1.4 \mathrm{~V}$ applied voltage), and (c) modulated layer with permittivity close to ENZ conditions $(\mathrm{B})\left(\left|\varepsilon_{\text {real }}\right|<0.5\right.$ at $\left.2-2.2 \mathrm{~V}\right)$, where strong field confinement occur in the active layer. ( $\mathrm{d}$ and e) The enlarged region focusing on the active layer in the left-hand edge of the gap. For clarification on the active layer, the vertical and horizontal axes are not shown in the same scale in (d) and (e). 
To gain further insight into the modulation mechanism, we simulated the complex index and modal structure for different applied voltages. The change in accumulation layer carrier concentration was determined from solutions of Poisson's equation with different applied biases. The Debye length of the structure was calculated to be $\sim 1 \mathrm{~nm}$, which is consistent with other reports for highly doped ITO., ${ }^{91}$ Using this value, the change in the complex refractive index of the plasmonic mode in the waveguide was calculated at different applied biases using electromagnetic simulations in which the index change in accumulation was approximated by a thin accumulation layer with homogeneous but enhanced refractive index. ${ }^{8,43}$ The simulated modulation strength of $\sim 1.36 \mathrm{~dB} / \mu \mathrm{m}$ at $2.2 \mathrm{~V}$ is shown in black in Figure 4a, corresponding to a complex effective index change of the plasmonic mode of $\Delta n=0.09+$ $0.0379 \mathrm{i}$ (corresponding maximum carrier concentration of 6.72 $\left.\times 10^{20} \mathrm{~cm}^{-3}\right)$. We note that at an applied voltage between 2 and $2.2 \mathrm{~V}$, the real part of the permittivity of the accumulation layer approaches zero (inset of Figure 4a). Because the normal component of the electrical displacement must be continuous with the adjacent material $\left(\varepsilon_{1} E_{1}=\varepsilon_{2} E_{2}\right),{ }^{34,44}$ a large electricfield enhancement occurs in the waveguide active layer (Figure $4 c, e)$. The highly confined field near a plasmon resonance together with the nonzero imaginary part of the active layer permittivity result in high absorption compared with nonresonant excitation (Figure $4 \mathrm{~b}, \mathrm{~d}$ ). ${ }^{34,43}$ Thus, a high on-off modulation ratio is expected from simple considerations, which agrees with our measurements (Figure 4a). We attribute the discrepancy between the measured and simulated modulation strengths to a systematic underestimation of the accumulation layer thickness, fabrication imperfection (e.g., surface roughness), and slight difference between the simulated and experimental structures, which is a topic of further investigation. It should be noted that the modulation strength would be significantly enhanced by increasing the overlap between the optical field and the active conducting oxide layer. We simulated the device by reducing the slot width to $100 \mathrm{~nm}$ and found a 3-fold enhancement of modulation strength with a maximum of $4.2549 \mathrm{~dB} / \mu \mathrm{m}$ (at $\varepsilon_{\text {real }}=-0.0801$ ), showing the potential for further engineering an ultraefficient plasmonic modulator.

We note that parasitic gate and wiring capacitances are the primary limiting factors for designing modulators for ultralow energy consumption optical nanocircuits. ${ }^{45,46}$ The obtained high modulation strength suggests that a device length of 1-2 $\mu \mathrm{m}$ could be sufficient to obtain $3 \mathrm{~dB}$ modulation. We estimate the energy consumption as $1 / 4 C V^{2},{ }^{45}$ where $C$ is the capacitance of the device, and $V$ is the applied voltage. With the device length of $2 \mu \mathrm{m}$ and applied voltage of $2 \mathrm{~V}$, the energy consumption of the device is $\sim 4 \mathrm{fJ} /$ bit, demonstrating a reasonable power-delay product for the development of nanooptical components. ${ }^{8}$ In addition, the device operation speed ( 3 $\mathrm{dB}$ modulation bandwidth) is determined by the $\mathrm{RC}$ time constant. Note that the measurement range of the frequency response of the plasmostor was limited by the slow response of the InGaAs infrared camera used for far-field microscopy (see Supporting Information) (the measured response time of the device in an AC configuration is $\sim 0.3 \mathrm{kHz}$ ). However, with device lengths of order $2 \mu \mathrm{m}$ the gate capacitance is as low as 4 fF. Assuming a realistic contact and wiring resistance of $R=$ $1000 \Omega$, the circuit speed can exceed $250 \mathrm{GHz}$, if the device is not loaded by other parasitics (this simple estimate does not include other sources of signal delay resulting from the wiring and RF probe connections). In practice, the RC time constant of an actual modulator using the present device would be limited by the driver electronics and the wiring capacitance, whose typical achievable values are estimated to be $\sim 100$ $\mathrm{GHz}{ }^{47}$ We anticipate that the modulation characteristics of a device could be improved in specific circuit applications by using alternative high- $k$ gate dielectrics, or refined electric interconnects designed for high speed rf operation or by introducing a resonant structure along the waveguide. ${ }^{48}$

Methods. Fabrication of the Plasmonic Modulator. The plasmonic slot waveguides used in our experiments are fabricated with a lift-off patterning process. A $100 \mathrm{kV}$ electron beam lithography system (Leica EBPG-5000+) with pattern generator transferred the waveguide designs to the resist (PMMA thickness $\sim 500 \mathrm{~nm}$ ). A $200 \mathrm{~nm}$ thin Au film is then deposited on the polished quartz glass substrate using e-beam evaporation. Standard procedures for development, lift-off, and manufacturer recipes are used for fabricating the waveguides. The slot's width and antenna dimensions are determined with scanning electron microscopy (SEM) after the lift-off process. The thin layer of $\mathrm{Al}_{2} \mathrm{O}_{3}$ is subsequently deposited onto the sample by ALD (Ultratech/Cambridge NanoTech). Finally, the ITO is sputtered selectively onto the waveguide array by using steel masks and a standard sputtering machine. For electric modulation experiments, all the waveguides are connected to external $300 \times 300 \mu \mathrm{m}^{2}$ gold pads, to which electrical contacts were wire bonded for applying a voltage (Figure 1e). Thin layers of $\mathrm{Al}_{2} \mathrm{O}_{3}$ and ITO were subsequently deposited on top of the waveguide system by ALD and sputtering as discussed.

ITO Sputtering. Sputtered $\left(\mathrm{In}_{2} \mathrm{O}_{3}\right) 0.9\left(\mathrm{SnO}_{2}\right) 0.1$ served as the active plasmonic material, with the $\mathrm{Au}$ and ITO layers serving as the electrical contacts (Figure 1e) for the modulator. For all experiments, the ITO was deposited using rf magnetron sputtering (ATC ORION, AJA International Inc.) in an oxygen/argon plasma (targets were purchased from Plasmaterials Inc.). All depositions were done at room temperature at a pressure of $3 \mathrm{mT}$ Torr. For the conducting oxide depositions, the gas combination during sputtering was $0.7 \% \mathrm{O}_{2}$ and $99.3 \% \mathrm{Ar}$, and the films were sputtered at a power of $50 \mathrm{~W}$. The electronic properties of the ITO were characterized by four-point probe and Hall measurements.

Cross-Polarization Far-Field Detection. A collimated and linearly polarized near-infrared laser $\left(\lambda_{0}=1550 \mathrm{~nm}\right)$ was focused using an $0.9 \times$ NA objective onto the nano-antenna to excite the gap-plasmon mode of the waveguide, and the waveguide output was imaged in reflection mode using an infrared InGaAs CCD camera. Since the waveguides were designed with a $90^{\circ}$ bend, the linearly polarized emission from the output of the waveguide is orthogonal to that of the input polarization, thus suppressing back-reflections coming from the incident beam, providing a good signal-to-noise ratio for the desired output signal. The absolute circuit transmission of the waveguides was determined by normalizing with the numericaperture-corrected spectral reflectivity of the ITO-air interface on the same sample. ${ }^{7}$

\section{ASSOCIATED CONTENT}

\section{S Supporting Information}

Experimental details on the measurement setup, calculation of dielectric constant of ITO, and IV measurements. This material is available free of charge via the Internet at http://pubs.acs.org. 


\section{AUTHOR INFORMATION}

\section{Corresponding Authors}

*E-mail: hwlee@caltech.edu.

*E-mail: haa@caltech.edu.

\section{Notes}

The authors declare no competing financial interest.

\section{ACKNOWLEDGMENTS}

This work was supported by the Multidisciplinary University Research Initiative Grant (Air Force Office of Scientific Research, FA9550-12-1-0024) and used facilities supported by the DOE 'Light-Material Interactions in Energy Conversion' Energy Frontier Research Center under grant DE-SC0001293 and the Kavli Nanoscience Institute (KNI) at Caltech. H.W.L. acknowledges the Croucher Foundation of Hong Kong. G.P. and S.P.B. acknowledge the National Science Foundation. A.K. and U.P. acknowledge funding of the German Research Foundation (DFG) through the Cluster of Excellence Engineering of Advanced Materials (EAM) and the Erlangen Graduate School in Advanced Optical Technologies (SAOT).

\section{REFERENCES}

(1) Zia, R.; Schuller, J. A.; Chandran, A.; Brongersma, M. L. Mater. Today 2006, 9, 20-27.

(2) Cai, W.; Shin, W.; Fan, S.; Brongersma, M. L. Adv. Mater. 2010, $22,5120-5124$

(3) Maier, S. A. IEEE J. Sel. Top. Quantum Electron. 2006, 12, 1671.

(4) Atwater, H. A. Sci. Am. 2007, 296, 56-63.

(5) Sorger, V. J.; Oulton, R. F.; Ma, R. M.; Zhang, X. MRS Bull. 2012, 37, 728-738.

(6) Brongersma, M. L.; Shalaev, V. M. Science 2010, 328, 440-441.

(7) Kriesch, A.; Burgos, S. P.; Ploss, D.; Pfeifer, H.; Atwater, H. A.; Peschel, U. Nano Lett. 2013, 13, 4539-4545.

(8) Miller, D. A. B. Proc. IEEE 2009, 97, 1166-1185.

(9) Melikyan, A.; Lindenmann, N.; Walheim, S.; Leufke, P. M.; Ulrich, S.; Ye, J.; Vincze, P.; Hahn, H.; Schimmel, T.; Koos, C.; Freude, W.; Leuthold, J. Opt. Express 2011, 19, 8855-8869.

(10) Dionne, J. A.; Diest, K.; Sweatlock, L. A.; Atwater, H. A. Nano Lett. 2009, 9, 897-902.

(11) Zhu, S.; Lo, G. Q.; Kwong, D. L. Appl. Phys. Lett. 2011, 99, 151114.

(12) Zhu, S.; Lo, G. Q.; Kwong, D. L. Opt. Express 2013, 21, 83208330.

(13) Joushaghani, A.; Kruger, B. A.; Paradis, S.; Alain, D.; Aitchison, J. S.; Poon, J. K. S. Appl. Phys. Lett. 2013, 102, 061101.

(14) Sorger, V. J.; Lanzillotti-Kimura, N. D.; Ma, R. M.; Zhang, X. Nanophotonics 2012, 1, 17-22.

(15) Dicken, M. J.; Sweatlock, L. A.; Pacifici, D.; Lezec, H. J.; Bhattacharya, K.; Atwater, H. A. Nano Lett. 2008, 9, 4048-4052.

(16) Melikyan, A.; Alloatti, L.; Muslija, A.; Hillerkuss, D.; Schindler, P. C.; Li, J.; Palmer, R.; Korn, D.; Muehlbrandt, S.; Thourhout, D. V.; Chen, B.; Dinu, R.; Sommer, M.; Koos, C.; Kohl, M.; Freude, W.; Leuthold, J. Nat. Photonics 2014, 8, 229-233.

(17) Papaioannou, S.; Kalavrouziotis, D.; Vyrsokinos, K.; Weeber, J.; Hassan, K.; Markey, L.; Dereux, A.; Kumar, A.; Bozhevolnyi, S. I.; Baus, M.; Tekin, T.; Apostolopoulos, D.; Avramopoulos, H.; Pleros, N. Sci. Rep. 2012, 2, 652.

(18) Randhawa, S.; Lachèze, S.; Renger, J.; Bouhelier, A.; Lamaestre,

R. E. D.; Dereux, A.; Quidant, R. Opt. Express 2012, 20, 2354-2362.

(19) Kruger, B. A.; Joushaghani, A.; Poon, J. K. S. Opt. Express 2012, 20, 23598-23609.

(20) Ooi, K. J. A.; Bai, P.; Chu, H. S.; Ang, L. K. Nanophotonics 2013, 2, 13-19.

(21) Thomas, R.; Ikonić, Z.; Kelsall, R. W. IEEE J. Sel. Top. Quantum Electron. 2013, 19, 4601708.
(22) Zhu, S.; Lo, G. Q.; Kwong, D. L. Opt. Express 2013, 21, 1269912712.

(23) Cai, W.; White, J. S.; Brongersma, M. L. Nano Lett. 2009, 9, 4403-4411.

(24) Piao, X.; Yu, S.; Park, N. Opt. Express 2012, 20, 18994-18999.

(25) Lou, F.; Dai, D.; Thylen, L.; Wosinski, L. Opt. Express 2013, 21, 20041-20051.

(26) Sweatlock, L. A.; Diest, K. Opt. Express 2012, 20, 8700-8709.

(27) Boltasseva, A.; Atwater, H. A. Science 2011, 331, 290-291.

(28) Naik, G. V.; Shalaev, V. M.; Boltasseva, A. 2013, 25, 3264-3294.

(29) West, P. R.; Ishii, S.; Naik, G. V.; Emani, N. K.; Shalaev, V. M.; Boltasseva, A. Laser Photonics Rev. 2010, 4, 795-808.

(30) Feigenbaum, E.; Diest, K.; Atwater, H. A. Nano Lett. 2010, 10, 2111-2116.

(31) Krasavin, A. V.; Zayats, A. V. Phys. Rev. Lett. 2012, 109, 053901.

(32) Huang, C.; Lamond, R. J.; Pickus, S. K.; Li, Z. R.; Sorger, V. J. IEEE Photon. J. 2013, 5, 2202411.

(33) Babicheva, V. E.; Kinsey, N.; Naik, G. V.; Ferrera, M.; Lavrinenko, A. V.; Shalaev, V. M.; Boltasseva, A. Opt. Express 2013, 21, 27326-27337.

(34) Lu, Z.; Zhao, W.; Shi, K. IEEE Photon. J. 2012, 4, 735-740.

(35) Lin, H. C.; Ye, P. D.; Wilk, G. D. Appl. Phys. Lett. 2005, 87, 182904.

(36) Wen, J.; Banzer, P.; Kriesch, A.; Ploss, D.; Schmauss, B.; Peschel, U. Appl. Phys. Lett. 2011, 98, 101109.

(37) Palik, D. Handbook of Optical Constants of Solids III; Academic: San Diego, CA, 1998.

(38) Fleming, J. W. Appl. Opt. 1984, 23, 4486-4493.

(39) Michelotti, F.; Dominici, L.; Descrovi, E.; Danz, N.; Menchini, F. Opt. Lett. 2009, 34, 839-841.

(40) Kim, J. S.; Cacialli, F.; Cola, A.; Gigli, G.; Cingolani, R. Synth. Met. 2000, 111-112, 363-367.

(41) Coutts, T. J.; Young, D. L.; Li, X. MRS Bull. 2000, 25, 58-65.

(42) Yota, I.; Shen, H.; Ramanathan, R. Vac. Sci. Technol., A 2013, 31, $01 \mathrm{~A} 134$.

(43) Vasudev, A. P.; Kang, J.; Park, J.; Liu, X.; Brongersma, M. L. Opt. Express 2013, 21, 26387-26397.

(44) Alù, A.; Silveirinha, M. G.; Engheta, N. Phys. Rev. E 2008, 78, 016604.

(45) Miller, D. A. B. Int. J. Optoelectron. 1997, 11, 155-168.

(46) Miller, D. A. B. Opt. Express 2012, 20, A293-A308.

(47) Chen, G.; Chen, H.; Haurylau, M.; Nelson, N. A.; Albonesi, D. H.; Fauchet, P. M.; Friedman, E. G. Integration, VLSI J. 2007, 40, 434446.

(48) Lee, H. W.; Burgos, S. P.; Papadakis, G.; Atwater, H. A. Proceedings of Frontiers in Optics: The 97th OSA Annual Meeting and Exhibit/Laser Science XXIX, Orlando, FL, October 6-10, 2013; The Optical Society: Washington, DC, 2013. 